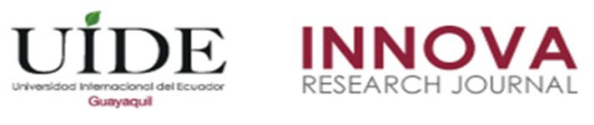

INNOVA Research Journal, ISSN 2477-9024

(julio 2016) Vol. 1, No.7 pp. 18-36

DOI: https://doi.org/10.33890/innova.v1.n7.2016.34

URL: http://revistas.uide.edu.ec/index.php/innova/index

Correo: innova@uide.edu.ec

\title{
PRODANALYSIS, un sistema para el aseguramiento de ingresos basado en minería de outliers
}

\section{PRODANALYSIS, a system for income assurance based on mining of outliers}

Gilberto Fernando Castro Aguilar

Iliana Pérez Pupo

Pedro Y. Piñero Pérez

Rosel Sosa

Pascual Verdecia Vicet

Universidad de las Ciencias Informáticas, Cuba

Mitchell Vásquez Bermúdez

Universidad Agraria del Ecuador, Ecuador

Autor para correspondencia: rsosag@uci.cu, iperez@uci.cu,ppp@uci.cu, gilberto.castroa@ug.edu.ec Fecha de recepción: 26 de junio de 2016 - Fecha de aceptación: 28 de julio de 2016

Resumen: El aseguramiento de ingresos es una de las técnicas que se aplica hoy desde las pequeñas hasta las grandes empresas para disminuir las pérdidas económicas por causa de fraudes, fugas o fallas en sus sistemas. Desde su inicio, el aseguramiento de ingresos ha ido aumentando su campo de aplicación y hoy es considerado como un campo inter-disciplinario que combina técnicas de estadística, bases de datos, inteligencia artificial, reconocimiento de patrones y minería de datos. En este trabajo se presenta un sistema que incluye facilidades para el aseguramiento de ingresos. El sistema llamado PRODanalysis 15.05 incluye técnicas de softcomputing que permiten el tratamiento de la imprecisión y la vaguedad en los conceptos para la detección automática de anomalías ante la prevención de posi-bles fraudes, fugas o fallas en el sistema. Los datos analizados corresponden a los dos entornos donde ha sido apli-cada la plataforma: en uno los datos se corresponden a los registros de llamadas telefónicas de la Empresa Nacional de Telecomunicaciones de Ecuador; en otro se corresponde a los datos registrados de los proyectos que fueron montados en la plataforma para su gestión.

Palabras Clave: aseguramiento de ingresos; dirección integrada de proyectos; softcomputing, prodanalysis

\begin{abstract}
Revenue assurance techniques are applied since a litter from big companies to solve different situations such as: fraud detection, revenue leakage, revenue loss, churn minimization, billing systems integration and service cost savings. Since beginning, revenues assurance scope has been increase, today is a multidisciplinary field that combine different techniques of artificial intelligence, statistical, soft computing, data mine and outliers mine. In this work we proposed a platform PRODanalysis 15.05 in order to improve revenue assurance process in TELCOS and Project Management oriented organizations. This platform includes soft computing techniques that permit the pre-vention of possible outliers like fraud, fugue and failures. The analyzed data corresponded to two environments that suit was applied: Telecommunication National Corporation CNT EP of Ecuador and project's data from Laboratory of Investigations of Project Management.
\end{abstract}

Key words: revenue assurance; project management; soft computing; prodanalysis 


\section{Introducción}

El aseguramiento de ingresos como área de conocimiento surge desde finales de la década de los 70' en el sector de las telecomunicaciones como disciplina orientada a la protección y recuperación de los recursos financieros de las organizaciones. Se extiende por su aplicabilidad a disímiles áreas del desarrollo social entre los que destacan la salud, la agricultura, la gobernabilidad y la gestión de organizaciones orientadas a proyectos. Varios autores han propuesto definiciones de esta disciplina, como en el caso de Khan (2014) donde se plantea que "el aseguramiento de ingresos es el conjunto de actividades que son aplicadas para asegurar que los procesos del negocio, la estructura organizacional, los controles y los sistemas de información, relacionadas con el ciclo de ingresos de las organizaciones, trabajen juntos con efectividad". En este concepto los autores reflejan con claridad la unidad indisoluble entre los procesos de aseguramiento de ingresos y los procesos de las organizaciones objeto de su aplicación.

Cuando se refiere a la efectividad se expresa la necesidad de que los resultados que se obtengan sean tangibles y verdaderos (Massyn, 2010) (Acosta, 2008). Según Mattison (2009)entre las técnicas más empleadas en el aseguramiento de ingresos se encuentran: el análisis de riesgos, análisis de intercambio, análisis de procesos, análisis de sistemas y el análisis estadístico.

Por otra parte dentro de la minería de datos se identifica un área particular conocida como minería de outliers(Ben-Gal I, 2005) que se dedica a la detección de datos anómalos en muestras de datos con aplicaciones en disímiles áreas (Karanjit \& Shuchita , 2012) entre las que destacan las telecomunicaciones, la salud entre otros.

En este trabajo se presenta una plataforma para el aseguramiento de ingresos. La misma está formada por dos elementos fundamentales: un sistema informático donde se incluye algoritmos orientados al aseguramiento de ingresos basados en técnicas de softcomputing y minería de outliers y un paquete de servicios para la formación y capacitación de personal para adquirir las competencias necesarios con este fin.

El trabajo está organizado de la siguiente forma. En la sección 2 contenido se distribuye en tres subsecciones: la 2.1 donde se realiza un pequeño estudio del estado del arte de aseguramiento de ingresos y de las técnicas de minería de outliers, la subsección 2.2 presenta la propuesta y la subsección 2.3 analiza los resultados obtenidos de aplicación de la propuesta en dos escenarios concretos. Luego en la sección 3 se discuten las conclusiones.

\section{Contenido}

\section{Breve estudio de aseguramiento de ingresos}

En sus inicios las actividades relacionadas con el aseguramiento de ingresos estaban concentradas en las empresas de telecomunicaciones y específicamente en el Director de Operaciones de Facturación (DOF) (TM Forum, 2015) (TM Forum, 2015). Pero esta concentración de actividades afectaba la eficiencia de los procesos y la ejecución efectiva de los procesos operacionales. 
Esta situación conllevó a la necesidad de formación de especialistas y a la creación de estándares.

En este contexto surge la Asociación Global de Profesionales de Aseguramiento de Ingresos (GRAPA) (Mattison, 2009), fundada con el objetivo de definir y estandarizar el trabajo de aseguramiento de ingresos.

Así mismo se crea el espacio de discusión de expertos en aseguramiento de ingresos TMForum(TM Forum, 2015)(TM Forum, 2015). TMFROM juega un papel destacado en el desarrollo de modelos de evaluación de madurez de las organizaciones respecto al aseguramiento de ingresos.

La mayoría de los autores (TM Forum, 2015) (Mattison, 2009) coinciden en la necesidad de la identificación de los factores que tienen mayor influencia en el aseguramiento de ingresos y arribaron a la siguiente distribución, figura 1.

Como se puede apreciar se identifican entre los factores fundamentales la aplicación de los estándares, las competencias del personal, la capacitación del personal y la integración de los procesos de aseguramiento de ingresos con los procesos organizacionales.

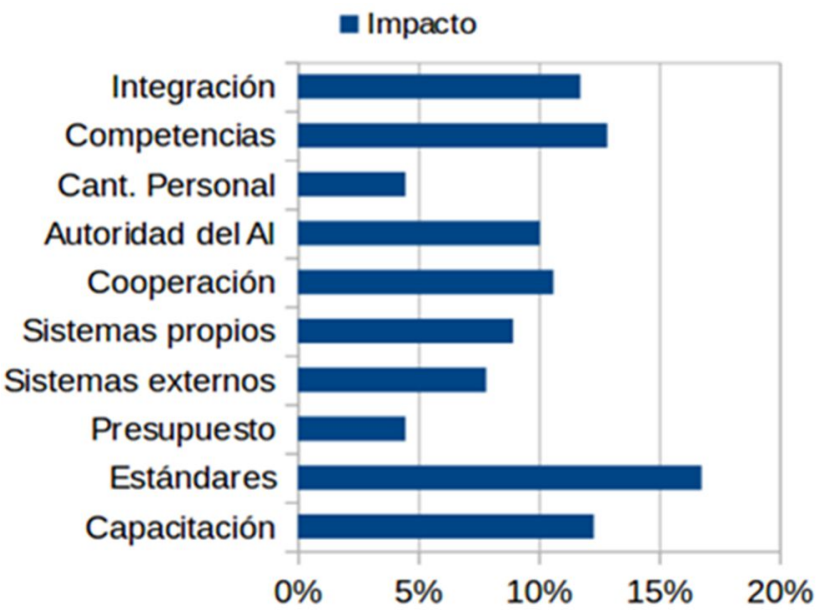

Figura 1: Impacto de las principales necesidades del Aseguramiento de Ingresos

El análisis de estos mismos factores sirvió a TMForum para promover su modelo de evaluación de la madurez de las organizaciones respecto a los procesos de aseguramiento de ingresos. Este modelo tiene 5 niveles: inicial, repetible, definido, manejado y optimizado. Y aunque es un gran paso de avance, se presenta como una guía abstracta que no dice cómo implementar completamente las mejores prácticas o herramientas.

Respecto a las herramientas más empleadas estas se comportan como muestra la tabla I.

Tabla I: Técnicas más empleadas por procesos (Mattison, 2009) 


\begin{tabular}{|c|c|}
\hline Proceso de análisis de riesgos & $\begin{array}{l}\text { Muestreo, Análisis de grupo, Análisis de distribución, Análisis tendencia central, } \\
\text { Regresión }\end{array}$ \\
\hline $\begin{array}{l}\text { Proceso de análisis de causa } \\
\text { raíz }\end{array}$ & Análisis de grupo, Análisis de distribución, Análisis tendencia central \\
\hline $\begin{array}{l}\text { Proceso de detección } \\
\text { temprana }\end{array}$ & Muestreo, Análisis de grupo, Chaid/Cart, RNA, \\
\hline Proceso de pronóstico & Muestreo, Análisis de grupo, Chaid/Cart, RNA, Regresión \\
\hline $\begin{array}{l}\text { Proceso de diseño de } \\
\text { controles }\end{array}$ & Muestreo, Chaid/Cart, Análisis de distribución, Análisis tendencia central \\
\hline
\end{tabular}

Como se puede ver la mayoría de las herramientas empleadas son basadas en modelos estadísticos tradicionales. Se identifica en este escenario la necesidad de técnicas que posibiliten el tratamiento de la imprecisión, la inconsistencia y la incertidumbre de los datos de origen tomados para el análisis. Se identifica además la necesidad de combinación de las técnicas estadísticas tradicionalmente empleadas con otras técnicas de análisis de datos como las encontradas en la disciplina de minería de outliers.

Conclusiones parciales

Se identifica por la mayoría de los autores que la aplicación de los procesos de aseguramiento de ingresos está indisolublemente ligada a los procesos de negocio de las organizaciones objeto de su aplicación.

Se identifica como tendencia la necesidad de combinar las estrategias reactivas, con estrategias activas que disminuyan el tiempo de detección de las fugas de ingresos y con estrategias proactivas orientadas a prevenir acciones de fallas, fraudes entre otras situaciones anómalas.

La mayoría de los autores refieren que existe la necesidad de desarrollo de herramientas novedosas que se adapten al dinamismo de las nuevas tecnologías para garantizar una exitosa aplicación de técnicas de aseguramiento de ingresos.

Se identifica que en la implantación de procesos de aseguramiento de ingresos es preciso el desarrollo de actividades de formación en los principales estándares, en las tecnologías y en los sistemas de información que registran los datos de los procesos organizacionales.

Breve análisis de la minería de outliers

Se presentan a continuación diferentes definiciones de outliers, propuestas por varios autores que ayudarán a entender mejor esta área del conocimiento y su posible relación con el aseguramiento de ingresos:

Hawkins define outlier como una observación que se desvía mucho del resto de las observaciones apareciendo como una observación sospechosa que pudo ser generada por mecanismos diferentes al resto de los datos.

Barnet and Lewis definen a una observación outlier, como una observación que se desvía marcadamente de otros miembros de la muestra en la cual se encuentra.

Johnson define que un outlier es una observación en los datos que aparece como inconsistente con respecto al resto de los datos.

A partir de estos elementos identificamos esta área del conocimiento como un área que puede contribuir significativamente en el proceso de aseguramiento de ingresos, en particular en 
los enfoques reactivo y activo para la detección de posibles registros que reflejen fugas de dinero por conceptos de fraude, fallas de sistemas entre otros.

Algunas de las técnicas que se han implementado en esta área del conocimiento se basan en: técnicas de agrupamientos (Bay \& Schwabacher, 2003)(E. N. Sathishkumar \& K. Thangavel, 2015), métodos basados en distancias entre objetos (Bay \& Schwabacher, 2003)(Knorr E. M., 1999), modelos basados en la densidad (Breunig, Kriegel, Ng., \& Sander, 2000), modelos estadísticos de basados en la distribución (Jifu Zhang, Sulan Zhang, Kai H. Chang, \& Xiao Qin, 2014)(Motaz K. Saad \& Nabil M. Hewahi, 2009)y modelos basados en redes neuronales artificiales (Hawkins, He, Williams, \& Baxter, 2002). Algunos autores clasifican las técnicas para la detección de outliersen dos grupos, las técnicas paramétricas y las no paramétricas como se muestra en la figura 2.

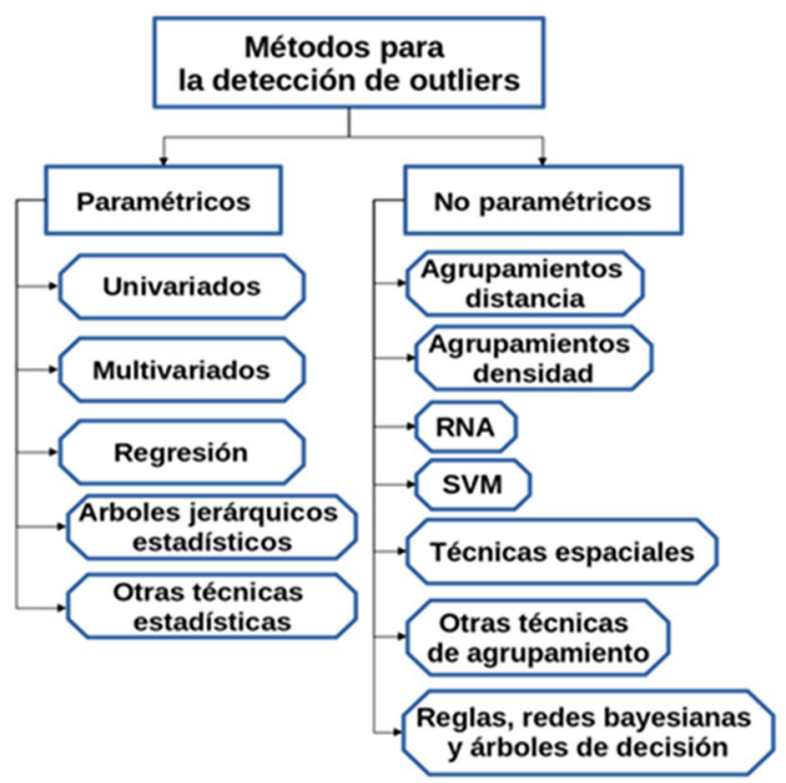

Figura 2: Clasificación de las técnicas de detección de outliers

Existen herramientas que incluyen técnicas de detección de outliers. Entre ellas se destacan la Biblioteca R (2015), con los paquetes (“outliers” y “outlierD”), SAS (2015), RapidMiner (2015) y Oracle datamine(Oracle, 2015). Sin embargo, la tendencia de estas herramientas es la aplicación de estrategias reactivas (trabajo ofline). De ahí que se identifica como una oportunidad la adaptación de las técnicas para su empleo en tiempo real soportando enfoques activos.

Otro problema no resuelto es la alta dimensionalidad de los datos y el gran volumen de los mismos que imponen con frecuencia el reto del trabajo en entornos distribuidos de forma paralela minimizando el gasto de recursos de comunicación entre los entornos.

\section{Plataforma PRODanalysis aplicación en el aseguramiento de ingresos}

En esta sección se propone una plataforma que incluye funcionalidades para el aseguramiento de ingresos. 
La plataforma PRODanalysisestá basada en la plataforma R. Incluye módulos para la ayuda a la toma de decisiones con algoritmos para el aprendizaje, la clasificación, agrupamientos, la minería de datos, la minería de outliers, el análisis estadístico de datos, la construcción de mapas estadísticos, el aseguramiento de ingresos entre otros.Ver figura 3 una vista de la plataforma en la gestión de datos outliers en la detección de problemas en la Provincia de Pichincha de ecuador.
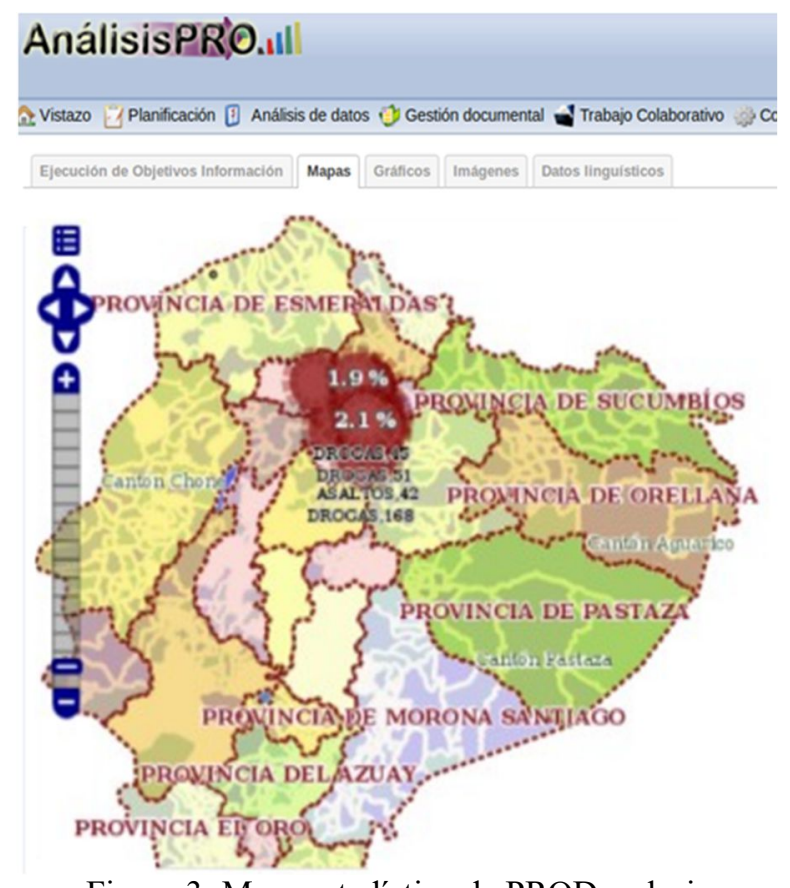

Figura 3: Mapa estadístico de PRODanalysis

Ver figura 4 aplicaciones de la plataforma en la evaluación de procesos eleccionarios en la formación de grupos, y las causas probables de decisión. 


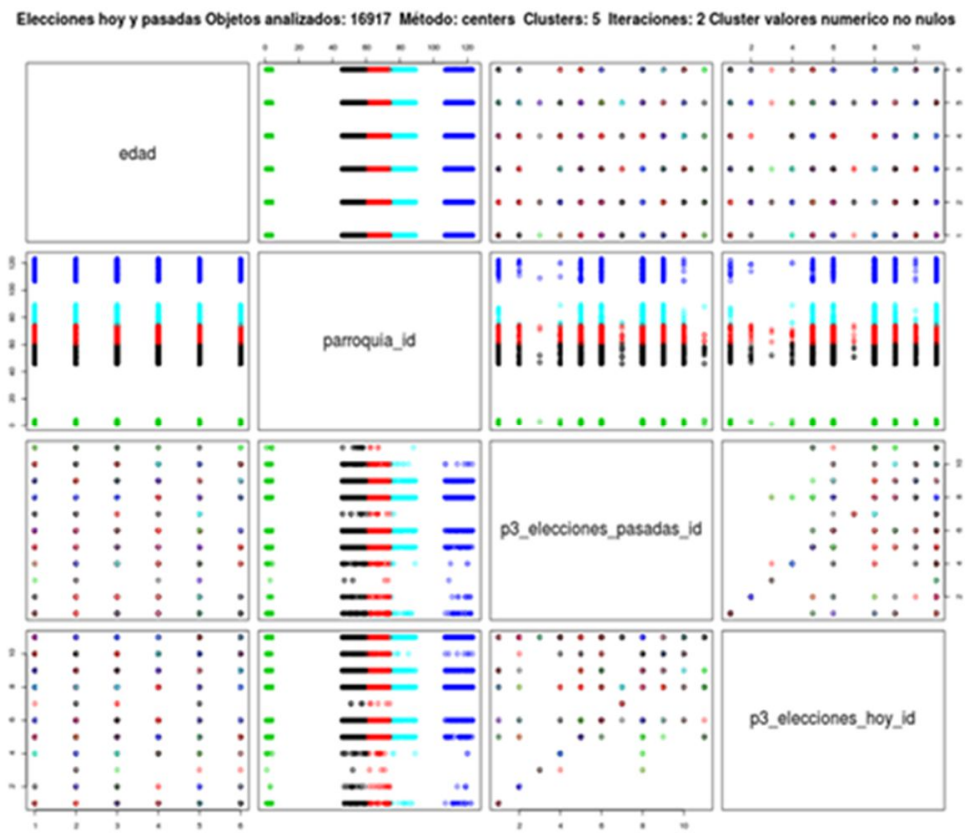

Figura 4: Vista plataforma PRODanalysis aplicada en cluster de datos de elecciones en Ecuador

Entre los algoritmos que incorpora la plataforma se encuentran:

- Algoritmos para la identificación de outliers a partir de la teoría de conjuntos aproximados.

- Algoritmos para la identificación de outliers a partir de los agrupamientos.

\section{¿Qué son los que se aplicaron en este trabajo?}

En particular para el aseguramiento de ingresos la plataforma propone se sigan los siguientes siete pasos como se explican en el resto de esta subsección.

\section{Paso 1. Planeamiento y diagnóstico}

Este paso comprende dos momentos. En un primer momento se debe comprender la fuente de datos y el proceso de la organización, para definir una taxonomía que ayude a identificar las situaciones que afecten el aseguramiento de ingresos, como posibles causas de fallos, fraudes o fugas. En un segundo momento se deben clasificar los interesados teniendo en cuenta el impacto e interés en el proceso del aseguramiento de ingresos de la organización.

\section{Paso 2. Pre-procesamiento de datos}

En este paso de agrupan las diferentes actividades relacionadas con el pre-procesamiento de los datos como la limpieza, estandarización y la selección de los atributos (tanto descriptores como decisores) que conformarán el sistema de información para el siguiente paso.

\section{Paso 3. Identificación de posibles outliers aplicando conjuntos aproximados}


En este paso se aplica como técnica para la identificación de outliers la teoría de conjuntos aproximados. Se muestra a continuación los conceptos fundamentales de la misma y como fueron aplicados en nuestra problemática.

La teoría de los conjuntos aproximados establece que dado un sistema de información definido como:

$S=(U, A \cup\{d\})$

Dónde:

U: es el universo de objetos,

A: es el conjunto de atributos de los objetos y

$\mathrm{d}$ : es el atributo decisor.

Se tiene $\mathrm{X} \subseteq \mathrm{U}$ y $\mathrm{B} \subseteq \mathrm{A}$.

A partir de estos elementos y la relación del atributo X respecto a B se pueden establecer los siguientes conjuntos de aproximaciones:

La aproximación inferior ver ecuación 1:

$$
\mathrm{B}^{*}(\mathrm{X})=\{\mathrm{x} \in \mathrm{U}: \mathrm{B}(\mathrm{x}) \subseteq \mathrm{X}\}
$$

Que agrupa a todos los objetos de los cuales no se tiene duda que pertenecen a determinada clase B.

La aproximación superior, ver ecuación 2:

$$
\mathrm{B}^{*}(\mathrm{X})=\{\mathrm{x} \in \mathrm{U}: \mathrm{B}(\mathrm{x}) \cap \mathrm{X}=\Phi\}
$$

Que recoge a todos aquellos que no pertenecen a la clase B o que no se está seguro de su pertenencia porque entran en inconsistencia con otros objetos de la clase.

La región frontera que contiene todos aquellos objetos que aparecen como inconsistentes porque a pesar de ser similares entre ellos pertenecen a clases diferentes. Estos objetos se dice que son indiscernibles respecto a los atributos considerados para su comparación y la función de comparación empleada, ver ecuación 3.

$$
\mathrm{BNB}(\mathrm{X})=\mathrm{B} *(\mathrm{X})-\mathrm{B} *(\mathrm{X})
$$

Aplicando estos conceptos al aseguramiento de ingresos se obtienen las siguientes situaciones. 
Los objetos de la aproximación inferior corresponden con los registros que no presentaron problemas o sea que se está seguro a que clase pertenecen y que deben ser registros normales.

Los objetos de la aproximación superior incluyen tanto objetos que pueden pertenecer a otras clases y ser normales como objetos que generan inconsistencias. Este conjunto es importante, pero para calcular el conjunto frontero.

Los objetos que pertenecen a la región frontera incluyen a todos los objetos que generan posibles inconsistencias. Entre estos objetos se encuentran los posibles outliers que representan en nuestro escenario registros anómalos que pueden ser provocados por acciones de fraude o fallas en los sistemas.

Claro que en esta región también hay registros normales que aparecen identificados como posibles outliers porque hay otros registros que son los verdaderos outliers que intentan ocultarse o confundirse con los primeros.

Este paso de detección de outliers termina devolviendo la región frontera por cada una de las clases y requiere de la siguiente fase para que termine de refinar cuál de los posibles objetos son los verdaderos outliers.

\section{Paso 4. Identificación de outliers aplicando agrupamiento con k-medoids:}

En el paso anterior se realiza una primera iteración de la identificación de outliers. En este paso se recurre a una de las herramientas que incorpora la plataforma PRODanalysis, ver figura 5.

Este método es un método basado en distancias y tiene las siguientes características, en algunas de las cuales supera al k-means tradicional:

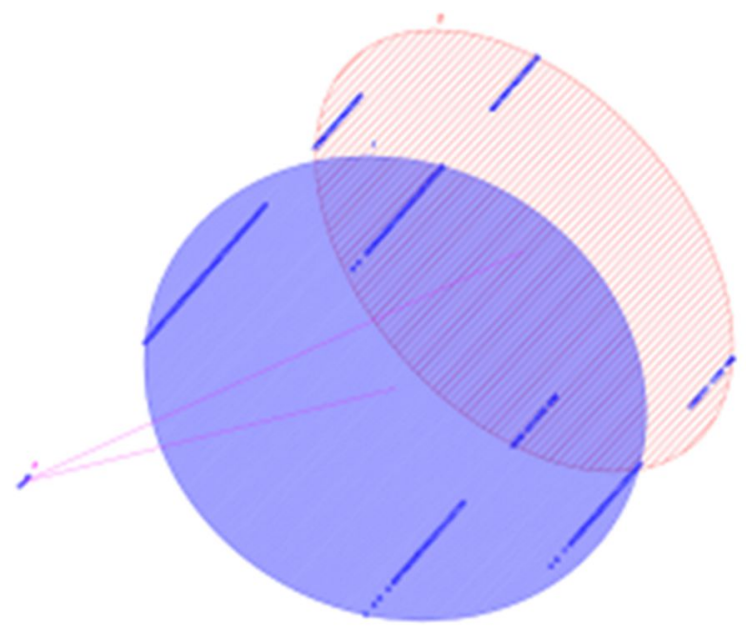

Figura 4: Ejemplo real de la plataforma PRODanalysis detección outliers

Es aplicable para el caso de los outliers y trabaja con matrices de disimilaridad. 
Es más robusto que el K-means porque minimiza la suma de las disimilaridades en lugar de la suma de la distancia euclidiana a diferencia del el k-means

Permite seleccionar el número de clusters manualmente o determinarlos, por sí mismo, considerando la media de las distancias.

Este algoritmo se basa en la determinación de los k objetos representativos (medoids ) de los diferentes grupos de objetos, identificando los mismos como aquellos objetos que minimicen las disimilaridades entre los objetos de un mismo grupo. Después de encontrar los k- medoids, el algoritmo construye alrededor de estos, los clusters de los objetos asignando a cada objeto al grupo cuyo medoid esté más cercano a él. Este proceso se repite hasta encontrar aquellos objetos representativos de cada grupo.Luego de esta fase aquellos objetos más alejados de todos los clusters pueden representar outliers.

\section{Paso 5. Verificación de los outlierscon un experto:}

En este paso se recurre a un experto para que sea quien valide si los registros clasificados outlierscomo resultado de la combinación de los pasos 4 y 5 son casos anómalos realmente. Además, se estudia la fuente de los datos del registro para identificar según la causa de la anomalía si se está en presencia de una fuga, un fallo o un fraude.

Toda esta información es computada para luego ser mostrada a través de un reporte en el paso 7 y último de este algoritmo.

\section{Paso 6. Computar el impacto económico:}

En este paso se estima el costo asociado a cada outliers identificado. Para ello se propone aplicar alguna de las siguientes técnicas:

- Estimación por analogía.

- Estimación de por tres valores.

- Estimación paramétrica.

- Juicio de expertos.

- Costo del aseguramiento de ingresos proactivo

La estimación de costos por analogía se basa en la información histórica y el juicio de expertos. Utiliza información del caso de análisis en cuestión como el teléfono, de origen de la llamada, la hora de ocurrencia, el periodo del año, el tipo del contrato, el tipo de tarifa horaria según el tipo de contrato y el impacto monetario de casos anteriores. Con todos estos elementos recupera los casos semejantes archivados en la base de datos y genera una posible respuesta a partir de la adaptación del nuevo caso considerando los valores de los casos similares recuperados.

Por lo general, la estimación de costos por analogía es menos costosa y requiere menos tiempo que las otras técnicas, pero también es menos exacta. Puede utilizarse en conjunto con 
otros métodos de estimación. La estimación análoga es más confiable cuando los casos recuperados son muy similares, no sólo en apariencia sino en los hechos.

Estimación por tres valores este método supone mejorar la exactitud de las estimaciones de costos tomando en consideración la incertidumbre. Para ello propone la estimación de tres valores:

- Costo más probable $(\mathrm{M})$ basado en una evaluación realista del experto.

- Costo optimista $(\mathrm{O})$ tomando como base el mejor escenario posible con el menor impacto posible.

- Costo pesimista (P) basado en el análisis del peor escenario o sea el mayor impacto posible.

Luego se procede a calcular el valor estimado a partir de la siguiente fórmula.

$$
\mathrm{ce}=(\mathrm{O}+4 \mathrm{M}+\mathrm{P}) / 6
$$

La estimación paramétrica utiliza una relación estadística entre los datos históricos y otras variables para calcular una estimación del impacto económico. Con esta técnica pueden lograrse niveles superiores de exactitud, dependiendo de la sofisticación y de los datos que utilice el modelo. La estimación paramétrica de costos puede aplicarse en conjunto con otros métodos de estimación.

Juicio de Expertos existen en la organización registros de casos anteriores, además los dinamismos de las organizaciones hacen de esta técnica una de las más usadas. Guiado por la información histórica, el juicio de expertos aporta una perspectiva valiosa sobre el ambiente y la información procedentes de casos similares anteriores. El juicio de expertos también puede utilizarse para determinar si es conveniente combinar métodos de estimación y cómo conciliar las diferencias entre ellos.

\section{Paso 7. Generar un reporte final:}

Generar reportes con la información asociada a cada outlier identificado, como:

- Estimación del costo asociado.

- Fuente de datos que lo generó.

- Clasificación del outlier en cuanto al tipo (puntual, contextual o colectivo) y en cuanto a su naturaleza (fuga, falla, fraude).

- Estado final de los ingresos: ingresos perdidos (por concepto de estimación del costo total estimado de los outliers) y el ingreso estable (la cantidad que no tuvo afectaciones).

\section{Análisis de los resultados de aplicación de la plataforma}

El empleo de la plataforma fue validado a partir de dos experimentos: 
Experimento 1: el plugin de aseguramiento de ingresos fue integrado a la plataforma PRODanalysis 14.05, el cual, a partir de los datos que se registran desde otros módulos de la misma plataforma, se pudo identificar algunas irregularidades financieras en algunos de los proyectos archivados en la base de datos de proyectos del Laboratorio de Investigaciones en Gestión de Proyectos.

Experimento 2: fue discutido el algoritmo implementado en el módulo de aseguramiento de ingresos propuesto con algunos expertos y ellos dieron sus consideraciones acerca del mismo. Fueron computadas las opiniones de los expertos usando técnicas de computación con palabras y consolidado con el método criterio de expertos.

\section{Resultados del experimento 1}

Para este experimento se integró la plataforma PRODanalysis 14.05 a la base de datos de proyectos terminados de la plataforma GESPRO 15.05 (Piñero, 2010) (Piñero P., Torres S., Izquierdo M., \& et al. GESPRO, 2013). Esta base de datos guarda información de los proyectos almacenando datos relacionados con el costo, órdenes de compra, logística, proveedores, ingresos, interesados, tareas, riesgos, resultados de los procesos de calidad, y toma de decisiones de los proyectos.

Al aplicar el algoritmo propuesto fueron detectados los siguientes casos:

- Algunas facturas enviadas por los proveedores estaban sobregiradas en costo.

- El alcance de los proyectos fue en algunos casos modificado extendiendo el mismo con nuevas funcionalidades, sin que esto fuera analizado para reajustes en la contratación. Provocando costos no previstos y un aumento no conciliado de los costos de ejecución internos.

- Algunas tareas se planifican con más tiempo del que necesitan implicando un alto costo innecesariamente.

- Algunos proyectos fueron ser detenidos temporalmente por causa de agentes externo como eventos naturales o por otra índole provocando periodos más largos de desarrollo con mayores costos para el proyecto por cuestiones de salarios.

El resumen de los costos identificados en los proyectos analizados y las causas asociadas a la pérdida de ingresos en los mismos se muestra en la tabla II.

Tabla II: Causas de fuga de ingresos en experimento 1

\begin{tabular}{ll}
\hline Facturas con sobre costo & $\$ 1500$ \\
Modificación del alcance de un proyecto & $\$ 1500$ \\
Sobre planeación de tareas de alto costo & $\$ 300$ \\
Detener un proyecto por factores externos & $\$ 2000$ \\
Total & $\$ 5300$ \\
\hline
\end{tabular}


En este experimento se usaron solo estrategias reactivas de aseguramiento de ingresos. Finalmente se estima que hubo una pérdida en los ingresos de aproximadamente \$5300.

\section{Resultados del experimento 2}

En este experimento se toman diferentes criterios acerca del módulo para el aseguramiento de ingresos integrado a la plataforma PRODanalysis 14.05 por los miembros del departamento de aseguramiento de ingresos de las compañías de telecomunicaciones CNT de Ecuador (CNT, 2015) y expertos de TMFORUM. Se aplicó técnicas de computación con palabras para consolidar los criterios obtenidos de las entrevistas realizadas a los expertos.

Los expertos (miembros del departamento de aseguramiento de ingresos) evaluaron la propuesta considerando los siguientes criterios:

- Aplicabilidad de la propuesta en compañías de telecomunicaciones.

- Comprensibilidad de la propuesta.

- Usabilidad de la propuesta.

- Resultados de aceptación de la propuesta en sus compañías.

Las técnicas de computación con palabras fueron aplicadas en los siguientes 3 pasos:

1. Se define un conjunto básico de términos lingüísticos (LBTL) para la evaluación de los criterios con los siguientes términos. LBTL $=$ \{nada, muy bajo, bajo, medio, alto, muy alto, perfecto\}.

2. Los expertos evalúan cada criterio usando alguno de los términos lingüísticos ver tabla III.

Tabla III: Estructura de la Evaluación de los expertos para cada criterio

\begin{tabular}{cccc} 
Criterios & \multicolumn{3}{c}{ Expertos } \\
& $e_{1}$ & $\ldots$ & $e_{m}$ \\
& $x_{1}^{11}$ & $\ldots$ & $x_{1}^{1 m}$ \\
$\vdots$ & $\vdots$ & $\vdots$ & $\vdots$ \\
$c_{p}$ & $x_{1}^{p 1}$ & $\ldots$ & $x_{1}^{p m}$ \\
\hline
\end{tabular}

Se transforma la preferencia de los expertos en conjuntos borrosos basados en la variable lingüística de la figura 6.

Siguiendo el modelo 2-tuplas [24] de computación con palabras se agregan las evaluaciones de los expertos consolidando las mismas por cada criterio a evaluar. Ver ecuación 5

$$
x^{e}(x)=\left(\frac{1}{n} \sum_{i=1}^{n}{ }^{-1}\left(\left(s_{i}, \alpha_{i}\right)\right)\right)=\left(\frac{1}{n} \sum_{i=1}^{n} \beta_{i}\right)
$$




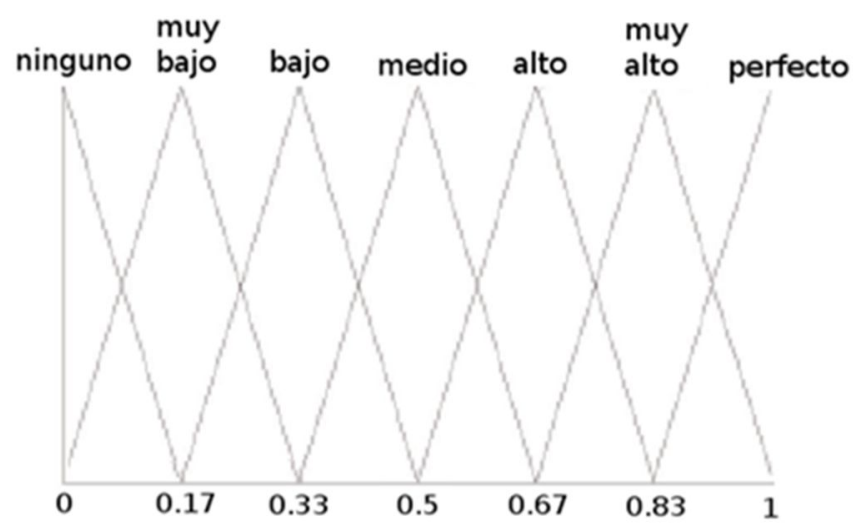

Figura 6: Variables lingüísticas usadas por los expertos para evaluar la propuesta

5. Finalmente se procede a analizar los resultados ver tabla IV

Tabla IV: Resultados de la evaluación

\begin{tabular}{|c|c|}
\hline Criterios & $\begin{array}{c}\text { Evaluación de los expertos } \\
\text { sumarizada }\end{array}$ \\
\hline $\begin{array}{l}\text { Aplicabilidad en compañías de } \\
\text { telecomunicaciones }\end{array}$ & Alta \\
\hline Comprensibilidad de la propuesta & Muy Alta \\
\hline Usabilidad de la propuesta & Alta \\
\hline $\begin{array}{l}\text { Resultados previos de la propuesta en sus } \\
\text { compañías }\end{array}$ & Media \\
\hline Agregación final & Alta \\
\hline
\end{tabular}

El criterio con menor evaluación fue "resultados previos de la propuesta en sus compañías" motivado por la etapa inicial de la investigación en la introducción de los resultados. El criterio mejor evaluado fue la comprensibilidad y la aplicabilidad de la propuesta. Este es un elemento positivo que muestra la aceptación de los expertos consultados para su posible aplicación en el escenario real. En general, la evaluación final dada por los expertos a la propuesta fue Alta.

\section{Conclusiones}

Se identificó como tendencia en las estrategias de aseguramiento de ingresos la necesidad de combinar las estrategias reactivas, con estrategias activas y proactivas, con el objetivo de disminuir los tiempos de detección de las fugas de ingresos y prevenir las posibles fallas o acciones de fraudes.

Se identifica que en la implantación de procesos de aseguramiento de ingresos es preciso el desarrollo de actividades de formación en los principales estándares, en las tecnologías y en los sistemas de información que registran los datos de los procesos organizacionales. 
La propuesta fue aplicada de forma experimental en una base de datos de proyectos terminados donde se identificaron diferentes situaciones que afectan los recursos financieros de la organización. En el caso de estudio se aplicó un método reactivo de aseguramiento de ingresos y se recomendó la introducción de la propuesta para su uso en una estrategia activa para el aseguramiento de ingresos. En el caso particular de aplicación se detectaron pérdidas en la organización por más de $\$ 5000$ unidades monetarias.

Se presentó la propuesta miembros del departamento de aseguramiento de ingresos de la Corporación de telecomunicaciones CNT de Ecuador y a expertos de aseguramiento de ingresos en TMFORUM, y fue evaluada positivamente respecto a la aplicabilidad y comprensibilidad de la misma.

Se aplicó el método 2-tuplas para lograr el consenso de los expertos demostrándose la aplicabilidad de este método. Se identifica la necesidad de realizar investigaciones futuras asociadas a combinar simultáneamente técnicas reactivas, pasivas y proactivas para el aseguramiento de ingresos.

\section{Bibliografía}

Azofra, V., \& Femández, A. (1992). Evolución reciente de la moderna teoría financiera. Anales de Ciencias Económicas y Empresariales, NQ7, 111-126.

Acosta Damas, M. (2009). Tesis de Doctorado. La entrevista en el sistema informativo de la televisión cubana: retos en la sociedad contemporánea. La Habana, La Habana, Cuba: Universidad de La Habana. Facultad de Comunicación Social.

Acosta, K. (2008). Aseguramiento de ingresos: una actividad fundamental en las empresas de telecomunicaciones. Vol. XXIX (No. 2). Revista Industrial.

Almenara, J. C. (15 de junio de 2002). La introducción del vídeo como instrumento de conocimiento en la enseñanza universitaria. Sevilla, Sevilla, España. Recuperado el 10 de agosto de 2015, de http://tecnologiaedu.us.es/revistaslibros/Artg-ice2.html

Aparicio, A. (2011). Recuperado el 23 de agosto de 2015, de https://www.uam.es/personal_pdi/stmaria/jmurillo/Met_Inves_Avan/Presentaciones/Cues tionario_\%28trab\%29.pdf

Bay, S., \& Schwabacher, M. (2003). Mining Distance-Based Outliers in Near Linear Time with Randomization and a Simple Pruning Rule. Proc. of The Ninth ACM SIGKDD International Conference on Knowledge Discovery and Data Mining.

Ben-Gal I. (2005). Outlier detection. Data Mining and Knowledge DiscoMuy Handbook: A Complete Guide for Practitioners and Researchers, Kluwer Academic Publishers, ISBN 0387-24435-2. Department of Industrial Engineering, Tel-Aviv University.

Bernal, C. A. (2010). Metodología de la investigación. Bogota: Pearson. 
Biblioteca R. (2015). Paquetes “outliers” y “outlierD” para la minería de outliers. http://cran.rproject.org/web/packages/outliers/outliers.pdf

Blank, L., \& Tarquin, A. (2002). Ingeniería Económica. México D.F.: McGraw Hill.

Breunig, M., Kriegel, H., Ng., R., \& Sander, J. (2000). LOF: Identifying density based local outliers. In: Proc. SIGMOD Conf, pp. 93-104.

Cabrero, J. (1989). Tecnología educativa: utilización didáctica del video. Barcelona.: PPU.

Calvo, E. (junio de 2011). Información audiovisual, multimedia y educación. 10. Granada., España.: Etc@net. Recuperado el 22 de septiembre de 2015, de http://www.ugr.es/ sevimeco/revistaeticanet/index.htm

Canet., F. (2009). Narrativa audiovisual: estrategias y recursos. Madrid: Síntesis.

CNT. (2015). Portal colaborativo, Departamento de Aseguramiento de Ingresos [en línea] Date Update on 2015, http://corporativo.cnt.gob.ec/cnt-ep-contribuye-con-el-estado/ Consultado octubre 2015.

Court Monteverde, E. (2012). Finanzas Corporativas. Buenos Aires: Cengage Learning.

Ezquerra, Á. (2010). Desarrollo audiovisual de contenidos científico-educativos. Enseñanza de las ciencias., 28 (3), 353-366.

E. N. Sathishkumar, \& K. Thangavel. (2015). A Novel Approach for Outlier Detection using Rough Entropy. Department of Computer Science Periyar University, In Wseas Transactions On Computers, E-ISSN: 2224-2872, Volume 14.

Damodaran, A. (2005). Valuation Approaches and Metrics: A Survey of the Theory. Hanover: Foundations and Trends in Finance.

Damodaran, A. (2001). The Dark Side of Valuation. Prentice Hall.

Fernández, M. C. (1997). Influencia del montaje en el lenguaje audiovisual. Madrid.: Libertarias.

Fernández, P. (1998). Valoración de Empresas. Barcelona: Gestión 200.

Fernández, P. (noviembre de 2008). IESE Business School. Recuperado el 30 de agosto de 2013, de Universidad de Navarra: http://ssrn.com/abstract=1266623

Fernández, P. (2008). Métodos de Valoración de Empresas. CIIF, 52.

Gallegos Muñoz, C., \& Medina Giacomozzi, A. (2011). Determinación del valor económico añadido: un modelo alternativo. Contabilidad y Negocios, 18. 
Hawkins, S., He, H., Williams, G., \& Baxter, R. (2002). Outlier detection using replicator neural networks. In Proc. of the Fifth Int. Conf. and Data Warehousing and Knowledge Discovery (DaWaK02).

Herrera F., \& L. Martınez. (2000). A 2-tuple fuzzy linguistic representation model for computing with words. IEEE Transactions on Fuzzy Systems, 8(6):746-752.

Jifu Zhang, Sulan Zhang, Kai H. Chang, \& Xiao Qin. (2014). An outlier mining algorithm based on constrained concept lattice. International Journal of Systems Science, vol. 45, no. 5, pp. 1170-1179, Impact Factor: 1.58, DOI: 10.1080/00207721.2012.745029 http://www.researchgate.net/publication/262317735.

Karanjit, S., \& Shuchita, U. (2012). Outlier Detection: Applications and Techniques. IJCSI International Journal of Computer Science Issues, Vol. 9, Issue 1, No 3, ISSN (Online): 1694-0814, www.IJCSI.org

Khan, N. (2014). Internship Report on Revenue Assurance and Fraud Management. (ID: 10104009).

Knorr E. M., N. (1999). Finding intensional knowledge of distance based outliers. In Proc. Of the 25th VLDB Conference, 211-222.

López Lubian, F., \& De Luna Butz, W. (2002). Finanzas Corporativas en la práctica. Madrid: McGraw Hill.

Massyn, R. (2010). A provisional taxonomy of revenue assurance: a grounded theory approach. (TH 621.3820683). (U. o. Johannesburg, Ed.) M.Sc. in Philosophy.

Marr, B., Roos, G., Neely, A., Pike, S., \& Gupta, O. (2004). Hacia la tercera generación en la medición de resultados. Revista de Contabilidad y Dirección , 1, 31-46.

Mattison, R. (2009). The Revenue Assurance Standards Release 2009. Published by XiT Press, Oakwood Hills, Illinois, USA, Copyright Rob Mattison 2009.

Mattison, R. (2009). The Revenue Assurance Standards Release 2009. Published by XiT Press, Oakwood Hills, Illinois, USA, Rob Mattison .

Medrano Samaniego, C. (2006). El poder educativo de la televisión. Revista de psicodidáctica, 11 (1), 93-108.

Milla Gutiérrez, A., \& Martínez Pedrós, D. (s.f.). Altair Consultores. Recuperado el agosto de 2013, de http://www.altairconsultores.com/images/stories/publicaciones/LIBRO_VALORACION.pdf 
Modigliani, F. y. (1958). The Cost of Capital, Corporation Finance and the Theory. American Economic Review, 261-297.

Morales, F. (2013). Montaje audiovisual: teoría, técnica y métodos de control. UOC.

Motaz K. Saad, \& Nabil M. Hewahi. (2009). A comparative Study of Outlier Mining and Class Outlier Mining. http://www.researchgate.net/publication/231521185

Oracle. (2015). Plataforma Oracle dataminer. Oracle, Update on 2015, Fecha de revisión octubre de 2015, http://docs.oracle.com/cd/B28359_01/datamine.111/b28129/intro_concepts.htm.

Palenzuela, V. A., \& Herrero, G. d. (2008). La huella indeleble de Modigliani y Miller: MM. Boletín de estudios económicos 63, 373-401.

Pandit, N. (diciembre de 1996). "La Creación de la teoría: una aplicación reciente del método puesto a tierra de la teoría". Recuperado el 3 de agosto de 2015, de http://www.nova.edu

Pereyra, T. M. (2008). Valoración de empresas: Una revisión de métodos actuales. Documento de trabajo No. 41, Facultad Administración y Ciencias Sociales, Universidad ORT Uruguay, 34.

Perona., A. M. (2010). Ensayo sobre video, documental y cine. Còrdova: Brujas.

Piñero P., Torres S., Izquierdo M., \& et al. GESPRO. (2013). Paquete para la gestión de proyectos. Revista Nueva Empresa, Cuba. Vol. 9, No. 1. pp. 45-53. ISSN: 1682-2455.

Piñero, P., (2010). Paquete de Herramientas para la Gestión de Proyectos. En Registro Centro Nacional de Registro de Derecho de Autor de Cuba, No Registro CENDA: 1540-2010, La Habana Cuba.

Portal, R., \& otros, y. (2008). Comunicación para el desarrollo: selección de lecturas. La Habana, La Habana, Cuba: Editorial Félix Varela.

Prieto Castillo, D. (2006). El interaprendizaje como clave de la educomunicación en mediaciones. Universidad Minuto de Dios (6).

Saladrigas Medina, H. (2005). Comunicación organizacional: Matrices teóricas y enfoques comunicativos. Revista Latina de Comunicación Social.

Sancho, J., Vilches, A., \& Gil, D. (2010). Los documentales científicos como instrumentos de educaciòn para la sostenibilidad. Eureka, 7 (3), 667-681.

Sandoval, T. (1998). Entrevista a "Mariano Cebrian comenta los últimos y los próximos cambios en comunicación audiovisual”. Revista Latina de Comunicación Social. (7). 
SAS. (2015). SAS Institute Inc. Update on 2015, Fecha de revisión octubre de 2015, https://www.sas.com/en_us/home.html

Stewart, G. (2000). En busca del valor. Barcelona: Gestión.

Ramos, J. L. (2002). ¿Qué es el vídeo educativo? Madrid, Madrid, España.

RapidMiner, I. (2015). Sistema RapidMiner. Headquarters, Update on 2015, Fecha de revisión octubre de 2015, https://rapidminer.com/, http://docs.rapidminer.com/

Romero, L. Á. (2010). Principios de contabilidad. McGraw Hill. Tipos de obras audiovisuales. (octubre de 2015). Cine y fotografía

TM Forum. (2015). Revenue Assurance practitioner blog: Do we need a new approach to revenue assurance in the digital world? \& Seeing is believing: Setting revenue assurance KPIs 2015.

TM Forum. (2015). Revenue Assurance a survey pre-result blog: Lack of cross-functional mandate holds back change, say Revenue Assurance professionals 2015.

Tostado, V. (1999). Manual de producción de video: un enfoque integral. Alhambra.

Wigodski, J. (14 de Julio de 2010). Metodología de la investigación. Recuperado el 19 de Julio de 2015, de http://metodologiaeninvestigacion.blogspot.com/2010/07/poblacion-ymuestra.html 